\title{
Shape and lattice deformation contributions to powder scattering
}

\author{
A. Leonardi ${ }^{1,2}, M$. Leoni ${ }^{3}$ \\ ${ }^{I}$ ISIS Neutron and Muon Facility, UKRI-Science and Technical Facility Council, Harwell Science Campus, Didcot, Oxfordshire \\ OX11 OYJ, UK, ${ }^{2}$ Institute for Multiscale Simulation, Friedrich-Alexander University Erlangen-Nürnberg, Cauerstrasse 3, 91058 \\ Erlangen (Germany), ${ }^{3}$ Saudi Aramco Research \& Development Center, PO box 62, 31311 Dhahran (Saudi Arabia)
}

\author{
alberto.leonardi@stfc.ac.uk
}

Important advancements in the field of powder X-ray scattering aim at improving the reach and accuracy of pattern analysis methods. Pair distribution function and other methods based on the Debye scattering equation (DSE) are the most straightforward as they directly compute the interference between couples of atoms. All interatomic distances for all grains in the powder have however to be computed. Whole powder pattern and pair distribution function modelling methods solve, instead, the same problem by considering whole objects in place of atoms. Even if not as general, they provide the same result but faster [1,2].

Common volume functions (CVFs) are used to model the scattering contribution from crystalline domains of any shape. However, CVFs are readily available for a limited set of regular shapes. Allegra and Wilson [3] computed the scattering line profile of deformed shapes applying a linear transformation. Although reprised multiple times, such approach is not suitable for techniques based on the CVF formalism. We generalized the paper of Allegra and Wilson considering the effects on the powder diffraction pattern of a deformation applied to the shape (i), to the underlying lattice (ii) or to both (iii).

Here we discuss the transformation of the CVF to account for the scattering contribution of domain shape and crystal structure deformations (Fig. A), and shape-structure relative orientation (Fig. B). As an example, starting from the CVF for a sphere (cube) domain and a cubic crystal structure, the transformation allows the analysis of (i) ellipsoid (parallelepiped) domains in a cubic lattice, (ii) sphere (cubic) domains in a triclinic lattice and (iii) ellipsoid (parallelepiped) domains in a homologous triclinic lattice. The presence of size and shape deformation dispersions are also considered. The resulting profiles are used to model both the intensity scattering contributions and the small-angle shape function used to correct numerical pair distribution function profiles. Finally, we analysed pattern simulated via DSE to assess the possibility of extracting the deformation information from real powder scattering data.
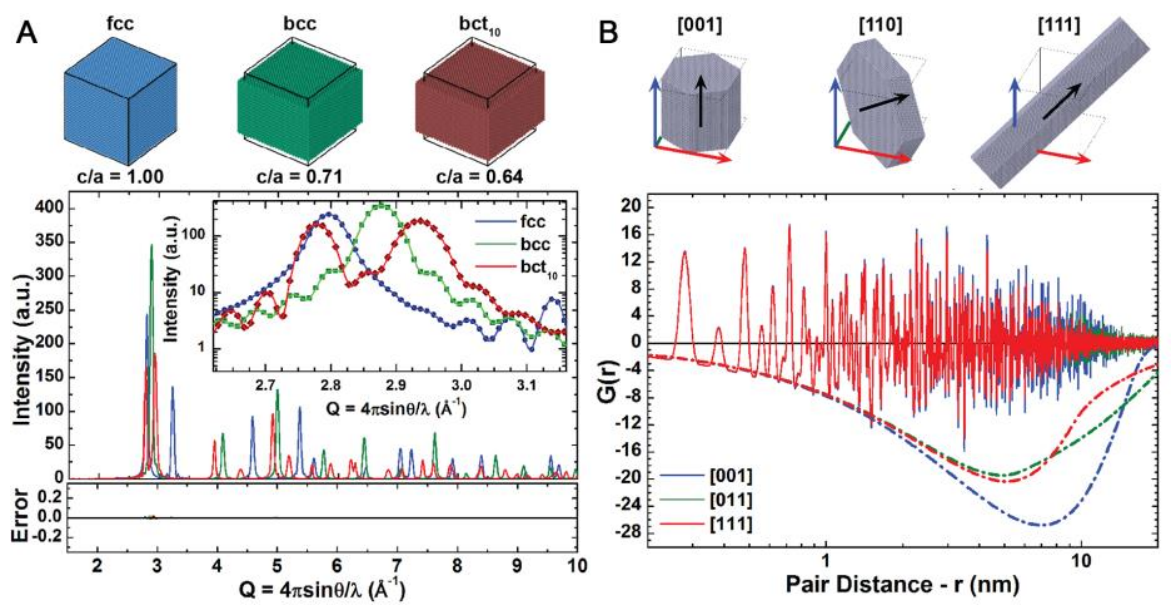

Figure 1. Dependence of intensity and pair distribution function profiles on the shape-structure deformation and relative orientation.

[1] Scardi, P. \& Leoni, M. (2002) Acta Crystallogr. A. 58, 190.

[2] Leonardi, A. (2021). IUCrJ 8, 257

[3] Allegra, G. \& Wilson, A. J. C. (1983) Acta Crystallogr. A. 39, 380.

Keywords: nanocrystals, lattice deformation, shape deformation, common volume function, shape function

A.L. wishes to acknowledge support from the Deutsche Forschungsgemeinschaft (grant No. LE4543/2-1).

Acta Cryst. (2021), A77, C841 